\section{Adaptação do Perfil de Saúde de Nottingham: um instrumento simples de avaliação da qualidade de vida}

\author{
Adaptation of the Nottingham Health Profile: \\ a simple measure to assess quality of life
}

\author{
${ }^{1}$ Departamento de \\ Fisioterapia, Universidade \\ Federal de Minas Gerais, \\ Belo Horizonte, Brasil. \\ 2 Departamento de Terapia \\ Ocupacional, Universidade \\ Federal de Minas Gerais, \\ Belo Horizonte, Brasil. \\ Correspondência \\ L.F. Teixeira-Salmela \\ Departamento de \\ Fisioterapia, Universidade \\ Federal de Minas Gerais. \\ Av. Antônio Carlos 6627, \\ Belo Horizonte, $M G$ \\ 31270-901, Brasil. \\ lfts@dedalus.lcc.ufmg.br
}

\begin{abstract}
The aim of the present study was to evaluate the psychometric properties of the Brazilian version of the Nottingham Health Profile (NHP), a generic instrument for quality of life assessment. This NHP was translated to Portuguese, following recommended methodology, and applied to 215 subjects, being 170 community elderly (66.3 \pm 8.5 years), 30 chronic stroke survivors (56.4 \pm 10.9 years), and 15 parkinsonians (59.6 \pm 10.2 years). The data was analysed by Rasch analysis. Out of the 38 items of NHP, only two items (5\%) did not fit the statistical model, indicating that the items collaborated to measure a unidimensional construct. However, some items of NHP were very easy for the elderly, stroke survivors and Parkinsonians, characterizing a ceiling effect, which could limit the use of the scale for individuals with higher levels of ability. The results, in spite of indicating that the instrument fits the Rasch statistical model for this sample, indicate a need for revision of the items, in order to make the questionnaire more clinically useful.
\end{abstract}

Quality of Life; Health Profile; Adaptation
Luci Fuscaldi Teixeira-Salmela 1

Lívia de Castro Magalhães ${ }^{2}$

Aline Cristina Souza 1

Maira de Castro Lima 1

Renata Cristina Magalhães Lima 1

Fátima Goulart 1

\section{Introdução}

Um instrumento de avaliação de qualidade de vida não deve se limitar, simplesmente, a medir a presença e a gravidade dos sintomas de uma doença. Deve também ser capaz de mostrar como as manifestações de uma doença ou tratamento são experimentadas pelo indivíduo $1,2,3,4$ valorizando aspectos da vida que geralmente não são considerados como "saúde", como, por exemplo, renda familiar, autonomia e trabalho 5 .

A utilização desses instrumentos na avaliação da qualidade de vida tem sido reconhecida como uma importante área do conhecimento científico no campo da saúde 2,3,6. Carr et al. 7 reafirmaram a importância destes instrumentos em inquéritos populacionais ou como identificadores das necessidades de saúde da população, bem como na prática clínica ou em experimentos clínicos controlados. Nestes tipos de estudos, é essencial o uso de medidas de qualidade de vida que sejam válidas, confiáveis e, sobretudo, sensíveis às mudanças clínicas obtidas com o tratamento 2,7. Em muitos países, devido ao acesso restrito a questionários, além dos gastos em tempo e dinheiro necessários para produzi-los, a adaptação de questionários tem sido vista como uma forma simples de se obter medidas válidas e confiáveis, visto que um questionário culturalmente adaptado indica que tanto a linguagem, quan- 
to o significado dos itens contidos no instrumento, são consistentes com aqueles do documento original $1,8,9,10$.

O Perfil de Saúde de Nottingham (PSN) é um instrumento genérico de avaliação de qualidade de vida, desenvolvido originalmente para avaliar a qualidade de vida em pacientes portadores de doenças crônicas 11,12,13. Trata-se de um questionário auto-administrado, constituído de 38 itens, baseados na classificação de incapacidade descrita pela Organização Mundial da Saúde, com respostas no formato sim/não. Os itens estão organizados em seis categorias que englobam nível de energia, dor, reações emocionais, sono, interação social e habilidades físicas. Cada resposta positiva corresponde a um escore de um (1) e cada resposta negativa corresponde a um escore zero (0), perfazendo uma pontuação máxima de 38 . Utilizando uma linguagem de fácil interpretação, o PSN fornece uma medida simples da saúde física, social e emocional do indivíduo 12,14 , sendo considerado clinicamente válido para distinguir pacientes com diferentes níveis de disfunção e para detectar alterações importantes no quadro de saúde do paciente ao longo do tempo 15,16. Sua consistência interna varia entre 0,90 e 0,94 e a confiabilidade teste-reteste é boa $(r=0,75$ a $0,88) 7$ além de ser sensível para discriminar indivíduos saudáveis de pacientes com sintomas de fadiga 14

O PSN tem sido utilizado nos últimos anos para a avaliação de qualidade de vida em idosos 17,18 , portadores de hemiplegia 14 e parkinsonismo 19. Porém, como a grande maioria das escalas de avaliação de qualidade de vida, esse instrumento foi desenvolvido e escrito originalmente na língua inglesa. Assim, para sua aplicação em outras culturas é necessário que, além da tradução, seja feita uma adaptação transcultural e a avaliação das propriedades de medida do instrumento para a população a ser empregada, visto que apenas a tradução literal do instrumento não é o bastante para sua utilização em outros idiomas 9 .

Guillemin et al. 9 após uma revisão sistemática da literatura no período de 1966 a 1992 sobre adaptação transcultural de instrumentos que mensuravam qualidade de vida, propuseram um conjunto de instruções padronizadas para este procedimento. Segundo os autores, a adaptação transcultural apresenta dois componentes - a tradução do instrumento propriamente dita e a adaptação do instrumento. Isso implica na combinação da tradução literal das palavras e sentenças de um idioma para o outro, de um contexto cultural para o outro e na avaliação da qualidade da medida adaptada em termos de sensibilidade, validade aparente e de conteúdo, re-aplicabilidade e adequação de escalas 9

O objetivo desse trabalho foi, através da análise Rasch, avaliar as propriedades psicométricas da versão adaptada do PSN para indivíduos idosos e portadores de hemiplegia e doença de Parkinson, examinando, assim, as limitações do instrumento para, se necessário, propor a revisão de alguns itens, de forma a tornar a escala útil para o uso clínico em nosso meio.

\section{Metodologia}

\section{Participantes}

Participaram deste estudo 170 idosos assintomáticos, 15 indivíduos portadores de doença de Parkinson, de leve a moderadamente comprometidos de acordo com a escala de Hoehn e Yahr 20 e 30 indivíduos portadores de hemiplegia secundária a acidente vascular cerebral, com tempo de evolução acima de nove meses, sem déficits de compreensão e capazes de deambular independentemente com ou sem órteses de auxílio.

Os idosos foram todos recrutados na cidade de Belo Horizonte, Minas Gerais, Brasil, em vários projetos preventivos de terceira idade, tais como, Vale a Pena Viver, Projeto Maiorida$d e$, clubes e centros de convivência (SESC-Gameleira e SESC-Lagoinha). Os indivíduos portadores de parkinsonismo e hemiplegia foram recrutados a partir de projetos (pesquisa e extensão) de atividade física para indivíduos portadores de deficiência, desenvolvidos no Departamento de Fisioterapia da Universidade Federal de Minas Gerais (UFMG) e no Laboratório de Distúrbios do Movimento do Hospital das Clínicas da UFMG, respectivamente. Optou-se por incluir no estudo indivíduos com essas patologias pelo fato de que a literatura mostra uma utilização mais freqüente do PSN nesses casos, o que torna particularmente interessante a aplicação do questionário adaptado nesses indivíduos. Não houve restrição quanto ao sexo em nenhum grupo. O estudo obteve aprovação do Comitê de Ética em Pesquisa da UFMG e todos os participantes assinaram um Termo de Consentimento concordando com a divulgação dos dados da pesquisa.

\section{O instrumento}

Segundo recomendação de Guillemin et al. 9, o PSN foi traduzido, do ponto de vista semântico, cultural e conceitual, para a língua portuguesa, 
por duas tradutoras com domínio do inglês, idioma de origem do instrumento. As tradutoras estavam conscientes dos objetivos básicos do questionário, para que se obtivesse uma versão mais apropriada do material original. Nessa fase, itens que não faziam sentido quando traduzidos literalmente para o português, foram adaptados. Por exemplo, o item "I am feelling on edge" foi traduzido como "eu me sinto extremamente irritado" e acrescido da expressão "com os nervos à flor da pele", que corresponde melhor ao sentido original em inglês. Como não havia no questionário nenhum item que não se aplicasse à nossa cultura, não foram necessárias maiores alterações, além da tradução.

A retro-tradução foi feita por duas outras tradutoras fluentes em ambos os idiomas e que não tinham conhecimento prévio da intenção e conceitos do material. A versão final adaptada do instrumento (Tabela 1) foi elaborada por um comitê de juízes especialistas, a partir das traduções e retro-traduções obtidas. Esse comitê foi constituído por profissionais da área com domínio do tema da pesquisa e também fluentes nos dois idiomas.

\section{Procedimento}

Pelo fato de grande parte dos indivíduos estudados serem analfabetos ou semi-analfabetos, e a fim de evitar erros de interpretação devido à heterogeneidade dos graus de instrução da população avaliada, ao invés de auto-administrado, conforme o texto original, o questionário foi aplicado individualmente por examinadores treinados, que seguiram instruções padronizadas.

\section{A análise Rasch}

O modelo Rasch é um tipo de análise estatística que vem sendo muito usada para avaliar as qualidades psicométricas das escalas atuais de reabilitação. Este tipo de análise permite calibrar a dificuldade dos itens e o nível de habilidade dos pacientes em um mesmo contínuo linear simples, dividido em intervalos iguais, ou logits, ao longo dos quais cada item da escala e cada paciente são alinhados 18,21,22,23.

O pressuposto básico da análise Rasch é que, quanto mais capaz uma pessoa, maior a sua probabilidade de receber escores altos em todos os itens de uma escala (fáceis ou difíceis). Por outro lado, quanto mais fácil o item, maior a probabilidade de qualquer pessoa receber escore alto nesse item 22,24. Quando todos os itens de um teste atendem a essas expectativas, significa que o teste se enquadra no modelo de medida 24 e a probabilidade é de que indivíduos com maior competência no domínio de uma dada função tenham escores mais altos que aqueles com menor competência. Esses princípios, no entanto, só se aplicam se o conjunto de itens medir uma habilidade unidimensional 25,26.

Programas computadorizados específicos para análise Rasch, como é o caso dos programas BIGSTEPS 27 calculam valores como a MnSq (goodness-of-fit) e o valor " $\mathrm{t}$ " associado a essa estimativa, que indicam se a relação entre a habilidade do indivíduo e a dificuldade do item atende aos pressupostos do modelo. Linacre \& Wright 27 sugeriram como valores razoáveis para sinalizar a adequação dos itens um $\mathrm{MnSq}=1,0 \pm 0,3$; com valor associado de $\mathrm{t}= \pm$ 2,0 . Um valor de MnSq muito alto indica que os escores nesse item foram muito variáveis ou erráticos. Em outras palavras, inesperadamente, pessoas menos capazes receberam escores altos nos itens difíceis 24 , o que sugere que, ou o item não combina com os outros para definir um contínuo de habilidade, ou existem problemas na definição do item, sendo necessária uma revisão do mesmo para que ocorra o enquadramento 21,24. Em contrapartida, um valor de MnSq muito baixo $(<0,7)$ indica pouca variabilidade de escores naquele item, ou seja, o padrão de resposta foi muito previsível ou determinista 21,24 . O primeiro resultado representa uma grande ameaça para a validade do teste, já o segundo, sinaliza que o item não discrimina pessoas com diferentes níveis funcionais.

Nesse estudo, a análise da calibração dos itens do PSN foi feita de três formas: (a) incluindo todos os indivíduos; (b) incluindo apenas os idosos assintomáticos e (c) incluindo apenas os portadores de hemiplegia e de doença de Parkinson. Como o escore errático indica maior problema na definição do item, foram assinalados para revisão os itens com valores de MnSq altos, em seus dois formatos, "Infit" e "Outfit", que sinalizam flutuações nas pontuações e a presença de escores extremos. Segundo critério utilizado na literatura 21 , quando mais de $5 \%$ do número total de itens do teste não se enquadram no modelo Rasch, isso é indicativo de que os itens da escala não combinam para medir um conceito unidimensional. Além disso, comparou-se a estabilidade dos itens quando usados para avaliar a qualidade de vida em idosos sadios versus idosos com incapacidades (hemiplegia e Parkinson) através do gráfico de dispersão. Se os valores são similares, espera-se como resultado uma linha de regressão diagonal. A estimativa de erro relacionada a cada item foi usada para traçar li- 
Perfil de Saúde de Nottingham (PSN).

\begin{tabular}{|c|c|c|}
\hline Itens & Não & Domínio \\
\hline 1. Eu fico cansado o tempo todo & & $\mathrm{NE}$ \\
\hline 2. Eu sinto dor à noite & & $\mathrm{D}$ \\
\hline 3. As coisas estão me deixando desanimado/deprimido(a) & & $\mathrm{RE}$ \\
\hline 4. A dor que eu sinto é insuportável & & $\mathrm{D}$ \\
\hline 5. Eu tomo remédios para dormir & & $\mathrm{S}$ \\
\hline 6. Eu esqueci como fazer coisas que me divertem & & $\mathrm{RE}$ \\
\hline 7. Eu me sinto extremamente irritado ("com os nervos à flor da pele") & & $\mathrm{RE}$ \\
\hline 8. Eu sinto dor para mudar de posição & & $\mathrm{D}$ \\
\hline 9. Eu me sinto sozinho & & IS \\
\hline 10. Eu consigo andar apenas dentro de casa & & $\mathrm{HF}$ \\
\hline 11. Eu tenho dificuldade para abaixar & & $\mathrm{HF}$ \\
\hline 12. Tudo para mim requer muito esforço & & $\mathrm{NE}$ \\
\hline 13. Eu acordo de madrugada e não pego mais no sono & & $\mathrm{S}$ \\
\hline 14. Eu não consigo andar & & $\mathrm{HF}$ \\
\hline 15. Eu acho difícil fazer contato com as pessoas & & IS \\
\hline 16. Os dias parecem muito longos & & RE \\
\hline 17. Eu tenho dificuldade para subir e descer escadas ou degraus & & $\mathrm{HF}$ \\
\hline 18. Eu tenho dificuldade para pegar coisas no alto & & $\mathrm{HF}$ \\
\hline 19. Eu sinto dor quando ando & & $\mathrm{D}$ \\
\hline 20. Ultimamente eu perco a paciência facilmente & & RE \\
\hline 21. Eu sinto que não há ninguém próximo em quem eu possa confiar & & IS \\
\hline 22. Eu fico acordado(a) a maior parte da noite & & $\mathrm{S}$ \\
\hline 23. Eu sinto como se estivesse perdendo o controle & & RE \\
\hline 24. Eu sinto dor quando fico de pé & & $\mathrm{D}$ \\
\hline 25. Eu acho difícil me vestir & & $\mathrm{HF}$ \\
\hline 26. Eu perco minha energia rapidamente & & NE \\
\hline $\begin{array}{l}\text { 27. Eu tenho dificuldade para permanecer de pé por muito tempo } \\
\text { (na pia da cozinha ou esperando o ônibus) }\end{array}$ & & $\mathrm{HF}$ \\
\hline 28. Eu sinto dor constantemente & & $\mathrm{D}$ \\
\hline 29. Eu levo muito tempo para pegar no sono & & $\mathrm{S}$ \\
\hline 30. Eu me sinto como um peso para as pessoas & & IS \\
\hline 31. As preocupações estão me mantendo acordado(a) à noite & & RE \\
\hline 32. Eu sinto que a vida não vale a pena ser vivida & & $\mathrm{RE}$ \\
\hline 33. Eu durmo mal à noite & & $\mathrm{S}$ \\
\hline 34. Eu estou tendo dificuldade em me relacionar com as pessoas & & IS \\
\hline 35. Eu preciso de ajuda para andar fora de casa (uma muleta, bengala ou alguém para $m$ & iar) & $\mathrm{HF}$ \\
\hline 36. Eu sinto dor para subir e descer escadas ou degraus & & $\mathrm{D}$ \\
\hline 37. Eu acordo me sentindo deprimido & & RE \\
\hline 38. Eu sinto dor quando estou sentado & & $\mathrm{D}$ \\
\hline
\end{tabular}

NE = Nível de energia; $D$ = Dor; RE = Reações emocionais; S = Sono; IS = Interação social; HF = Habilidades físicas A lista cita alguns problemas que as pessoas podem enfrentar no dia a dia. Por favor, leia cada item com atenção. Se o problema acontece com você, coloque um X abaixo da coluna "Sim". Se o problema não acontece com você, coloque um X abaixo da coluna "Não". Se você não estiver certo da resposta, pergunte a si mesmo se isso acontece com você na maioria das vezes. É importante que você responda a todas as questões 
nhas de controle de qualidade, que delimitam uma margem de diferença. Itens que se localizam fora das linhas de controle apresentam nível de dificuldade ou calibração diferente 26 .

A análise Rasch fornece ainda um índice de separação dos itens por nível de dificuldade e de indivíduos por nível de habilidade, o que nos permite ter uma estimativa dos níveis de habilidade dos indivíduos. Espera-se que um teste divida os pacientes em pelo menos três níveis de habilidade (baixo, médio e alto) 18.

\section{Resultados}

Fizeram parte do presente estudo um total de 215 indivíduos, sendo 170 idosos assintomáticos, com idade variando entre 60 e 83 anos $(66,3 \pm 8,5)$ dos quais $18,0 \%$ eram homens e $82,0 \%$ mulheres, 30 indivíduos portadores de hemiplegia crônica, sendo $56,7 \%$ homens e $43,3 \%$ mulheres, com idade entre 34 e 83 anos $(56,4 \pm 10,9)$ e 15 indivíduos portadores de doença de Parkinson leve, divididos em $66,7 \%$ homens e $33,3 \%$ mulheres, cuja idade variou entre 40 e 77 anos $(59,6 \pm 10,2)$. Desses indivíduos, $11,0 \%$ eram analfabetos, $52,0 \%$ estudaram apenas até a 4 a série do Ensino Fundamental. Do restante, $7,0 \%$ tinham o 1 o grau completo, $12,0 \%$ o 2 o grau completo e apenas $5,0 \%$ possuíam curso superior.

O resultado da análise Rasch para todos os indivíduos estudados, apresentado na Tabela 2 , indica que dos 38 itens do perfil apenas dois (o item de número 9 - "Eu me sinto sozinho" e 21 - "Eu sinto que não há ninguém próximo em quem eu possa confiar") não se encaixaram nas expectativas do modelo. Conclui-se, portanto, que os itens da escala se combinam para medir um conceito unidimensional. Observa-se que o item mais difícil (maior probabilidade de resposta sim) da escala foi o de número 27 - "Dificuldade para ficar de pé" e o mais fácil (maior probabilidade de resposta não) foi o item de número 14 - "Não consigo andar". O índice de separação dos pacientes foi de apenas 2,18 indicando que os itens dividem as pessoas em aproximadamente 2,5 níveis de habilidade.

A Figura 1 representa o mapa de itens para todos os indivíduos. Esse mapa de itens, baseado na calibração dos itens da escala, é uma ilustração do contínuo de habilidades dos pacientes, à esquerda, e do contínuo de dificuldade dos itens, à direita. Em síntese, essa é uma representação do desempenho das pessoas/pacientes em relação ao nível de dificuldade dos itens da escala. Através desse mapa, pode-se observar o grande número de indiví- duos no topo, sem itens para avaliá-los, fenômeno conhecido como efeito teto (ceiling ef$f e c t$ ). Na parte inferior do contínuo, observa-se que alguns itens foram muito fáceis, não havendo na amostragem indivíduos com tão pouca qualidade de vida.

$\mathrm{Na}$ análise incluindo apenas os dados dos idosos assintomáticos, encontrou-se um único item errático, o item de número 9 - "Eu me sinto sozinho". Na análise dos dados dos indivíduos portadores de hemiplegia e de doença de Parkinson, encontra-se um outro item errático, o item 5 - "Eu tomo remédios para dormir". Além disso, como apresentado na Figura 2, a calibração de itens para as duas amostragens foi diferente, já que oito itens apresentam dificuldades relativas diferentes num grupo em relação ao outro. As linhas de controle de qualidade foram traçadas tendo como base o erro de medida. Os itens 8,13 e 36, relacionados à dor e sono, localizados fora das curvas no lado direito do gráfico, são itens mais fáceis (isto é, maior número de respostas não) para os indivíduos portadores de alterações neurológicas. Os itens 25, 26, 30, 31,35 , relacionados à habilidade física, interação social, reação emocional e nível de energia, localizados acima ou à esquerda no gráfico, foram itens mais fáceis para idosos sadios.

\section{Discussão}

Os dados coletados a partir da aplicação da versão adaptada do PSN em idosos assintomáticos, hemiplégicos e parkinsonianos, foram submetidos à análise estatística, que acusou dois itens erráticos: o item 9 ("Eu me sinto sozinho"), e o item 21 ("Eu sinto que não há ninguém próximo em quem eu possa confiar"). Isso significa que as respostas a esses itens foram variáveis ou controversas, ou seja, inesperadamente, pessoas com uma boa qualidade de vida se sentem sozinhas, ou não têm em quem confiar. Talvez esse fato possa ser explicado por uma característica relativamente comum entre os idosos, com ou sem patologias associadas, que é a solidão e depressão 28 . Não é raro, nessa faixa etária, a perda de pessoas próximas e entes queridos, incluindo o próprio parceiro e os filhos, que se casam ou saem de casa. Tornase difícil para esses indivíduos encontrarem outro parceiro ou outras pessoas amigas, com as quais eles acreditem poder contar. Isso faz com que, mesmo que sejam indivíduos ativos fisicamente, com um bom nível de saúde e participação na comunidade, se sintam sozinhos, ou não tenham em quem confiar, justificando a variabilidade das respostas nesses itens. 
Calibração dos itens do Perfil de Saúde de Nottingham respondido por idosos, hemiplégicos e parkinsonianos.

\begin{tabular}{|c|c|c|c|c|c|c|}
\hline \multirow[t]{2}{*}{ Nível de dificuldade do item/item } & \multirow{2}{*}{$\begin{array}{c}\text { Medida } \\
\text { (calibração) }\end{array}$} & \multirow[t]{2}{*}{ Erro } & \multicolumn{2}{|c|}{ Infit } & \multicolumn{2}{|c|}{ Outfit } \\
\hline & & & $\mathrm{MnSq}^{*}$ & $\mathrm{t}$ & $\mathrm{MnSq}^{\star}$ & $\mathrm{t}$ \\
\hline \multicolumn{7}{|l|}{ Alto } \\
\hline 27. Dificuldade para ficar de pé & 1,45 & 0,17 & 0,98 & $-0,30$ & 0,92 & $-0,80$ \\
\hline 3. Me sinto desanimado/deprimido & 1,31 & 0,17 & 1,01 & 0,10 & 1,03 & 0,30 \\
\hline 17. Dificuldade subir/descer escadas & 1,08 & 0,17 & $0 ., 97$ & $-0,40$ & 0,93 & $-0,50$ \\
\hline 9. Me sinto sozinho & 1,02 & 0,17 & 1,29 & 3,70 & 1,54 & $3,70^{\star *}$ \\
\hline 13. Acordo e não pego no sono & 0,91 & 0,17 & 1,00 & 0,00 & 0,99 & $-0,10$ \\
\hline 20. Perco a paciência facilmente & 0,88 & 0,17 & 1,16 & 2,00 & 1,28 & 2,00 \\
\hline 11. Tenho dificuldades para abaixar & 0,63 & 0,18 & 1,09 & 1,10 & 1,06 & 0,40 \\
\hline 18. Acho difícil pegar coisas no alto & 0,60 & 0,18 & 1,01 & 0,10 & 1,00 & 0,00 \\
\hline 7. Estou com nervos à flor da pele & 0,54 & 0,18 & 1,00 & 0,00 & 1,06 & 0,40 \\
\hline 31. Preocupações não deixam dormir & 0,54 & 0,18 & 0,92 & $-1,00$ & 0,89 & $-0,70$ \\
\hline 29. Demoro a pegar no sono & 0,51 & 0,18 & 0,98 & $-0,20$ & 0,96 & $-0,20$ \\
\hline 24. Sinto dor quando fico de pé & 0,48 & 0,18 & 0,90 & $-1,20$ & 0,81 & $-1,30$ \\
\hline 36. Dor subir e descer escadas & 0,44 & 0,18 & 0,91 & $-1,10$ & 0,95 & $-0,30$ \\
\hline 2. Sinto dor à noite & 0,41 & 0,18 & 1,02 & 0,30 & 0,91 & $-0,50$ \\
\hline 19. Sinto dor quando ando & 0,31 & 0,18 & 0,88 & $-1,40$ & 0,79 & $-1,30$ \\
\hline 6. Não faço coisas que divertem & 0,21 & 0,19 & 1,20 & 2,00 & 1,38 & 1,80 \\
\hline 8. Sinto dor para mudar de posição & 0,21 & 0,19 & 0,91 & $-1,00$ & 0,77 & $-1,40$ \\
\hline 12. Tudo requer muito esforço & 0,07 & 0,19 & 0,79 & $-2,20$ & 0,75 & $-1,30$ \\
\hline 15. Difícil fazer contato com pessoas & $-0,01$ & 0,19 & 1,10 & 1,00 & 0,91 & $-0,40$ \\
\hline 16. Os dias parecem longos & $-0,04$ & 0,19 & 0,87 & $-1,30$ & 0,71 & $-1,50$ \\
\hline 23. Sinto estar perdendo o controle & $-0,04$ & 0,19 & 0,92 & $-0,80$ & 0,93 & $-0,30$ \\
\hline 26. Sinto que perco a energia rápido & $-0,04$ & 0,19 & 0,91 & $-0,90$ & 0,79 & $-1,00$ \\
\hline 33. Durmo mal à noite & $-0,08$ & 0,20 & 1,04 & 0,40 & 0,90 & $-0,50$ \\
\hline 22. Fico acordado à noite & $-0,16$ & 0,20 & 0,93 & $-0,60$ & 0,78 & $-1,00$ \\
\hline 30. Me sinto peso para as pessoas & $-0,16$ & 0,20 & 1,04 & 0,40 & 0,89 & $-0,50$ \\
\hline 34. Acho difícil me relacionar & $-0,16$ & 0,20 & 1,09 & 0,80 & 0,90 & $-0,40$ \\
\hline 21. Não tenho ninguém para confiar & $-0,24$ & 0,20 & 1,23 & 1,90 & 1,68 & $2,40^{\star *}$ \\
\hline 28. Sinto dor constante & $-0,24$ & 0,20 & 0,88 & $-1,10$ & 0,73 & $-1,20$ \\
\hline 5. Tomo remédios para dormir & $-0,41$ & 0,21 & 1,28 & 2,10 & 1,56 & 1,80 \\
\hline 37. Acordo deprimido & $-0,41$ & 0,21 & 0,81 & $-1,60$ & 0,67 & $-1,40$ \\
\hline 38. Sinto dor sentado & $-0,50$ & 0,21 & 1,01 & 0,10 & 1,25 & 0,80 \\
\hline 1. Fico cansado o tempo todo & $-0,55$ & 0,22 & 0,82 & $-1,50$ & 0,70 & $-1,20$ \\
\hline 25. Acho difícil me vestir & $-0,59$ & 0,22 & 0,94 & $-0,40$ & 0,95 & $-0,20$ \\
\hline 4. A dor que sinto é insuportável & $-0,69$ & 0,22 & 1,01 & 0,10 & 1,12 & 0,40 \\
\hline 35. Preciso de ajuda para andar fora de casa & $-1,02$ & 0,24 & 1,13 & 0,80 & 0,91 & $-0,20$ \\
\hline 32. A vida não vale a pena & $-1,21$ & 0,26 & 0,97 & $-0,20$ & 0,80 & $-0,50$ \\
\hline 10. Não consigo andar fora de casa & $-2,20$ & 0,36 & 0,98 & $-0,10$ & 1,51 & 0,70 \\
\hline 14. Não consigo andar & $-2,86$ & 0,47 & 1,08 & 0,20 & 1,00 & 0,00 \\
\hline Baixo & & & & & & \\
\hline
\end{tabular}

* $\mathrm{MnSq}=$ Mean Square

** Itens que não se enquadraram no modelo estatístico 
Analisando isoladamente os dados obtidos pela avaliação dos idosos assintomáticos, observou-se novamente o item de número 9 (" $E u$ me sinto sozinho"), apresentando um padrão variável ou inesperado de respostas, o que também poderia ser explicado pelo isolamento ou depressão 28. Ao analisar apenas os dados dos indivíduos portadores de hemiplegia e de doença de Parkinson, encontra-se, como item errático, apenas o item 5 ("Eu tomo remédios para dormir"). O esperado seria que indivíduos com uma boa qualidade de vida não usassem remédios para dormir, o que na verdade não aconteceu. Essa variação pode ter ocorrido pelas próprias características dos pacientes, indivíduos portadores de patologias crônicas, freqüentemente incapacitantes e fortemente ligadas à depressão, daí a alta freqüência de utilização de medicamentos para dormir, mesmo entre aqueles indivíduos classificados como tendo uma boa qualidade de vida 29 .

Comparando-se a calibração dos itens do questionário para os dois grupos que compõem a amostra, notaram-se oito itens com calibração diferente. Os itens 8 ("Eu sinto dor para mudar de posição"), 13 ("Eu acordo de madrugada e não pego mais no sono") e 36 ("Eu sinto dor para subir e descer escadas ou degraus"), são itens mais fáceis para hemiplégicos e parkinsonianos. Isso pode estar relacionado à freqüente utilização de medicamentos analgésicos e ansiolíticos ou antidepressivos 29 por esses indivíduos, discutida anteriormente, que contribui para o mascaramento da dor e sono, causando a impressão de facilidade nas tarefas que envolvam esses domínios. Já os itens 25 ("Eu acho difícil me vestir"), 26 ("Eu perco minha energia rapidamente"), 30 ("Eu me sinto como um peso para as pessoas"), 31 ("As preocupações estão me mantendo acordado(a) à noite") e 35 ("Eu preciso de ajuda para andar fora de casa - uma muleta, bengala ou alguém para me apoiar") se mostraram mais fáceis para idosos assintomáticos. Esse resultado condiz com o esperado, visto que esses itens estão ligados à dependência e depressão, e obviamente, se compararmos idosos assintomáticos, inseridos na comunidade, com indivíduos portadores de seqüelas motoras, os idosos assintomáticos são mais independentes e, possivelmente, apresentam menos queixas de depressão.

Analisando o mapa de itens, uma grande limitação da escala fica evidente. Observa-se o chamado efeito teto para indivíduos de habilidade mais alta, e, no outro extremo da escala, itens fáceis demais que não apresentaram dificuldade para nenhum dos participantes. Em outras palavras, não existem no questionário

\section{Figura 1}

Mapa representando o contínuo de dificuldade dos itens e habilidade indivíduos avaliados.

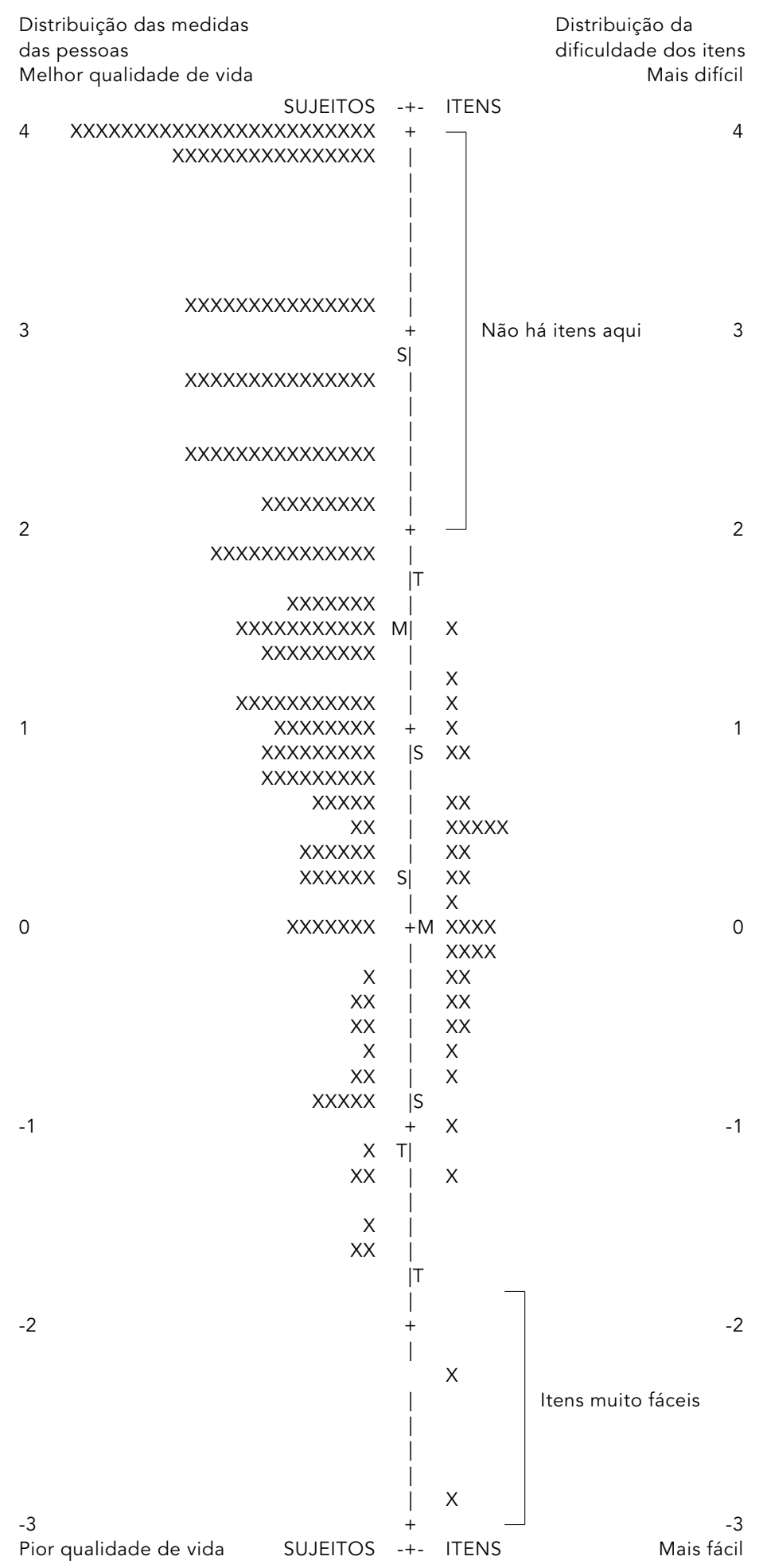

O mapa inclui todos os participantes do estudo.

Cada $\mathrm{X}$ a esquerda da linha central representa dois idosos,

cada $\mathrm{X}$ a direita da linha central representa um item do teste 


\section{Figura 2}

Comparação da calibração de itens para idosos, hemiplégicos e parakinsonianos.

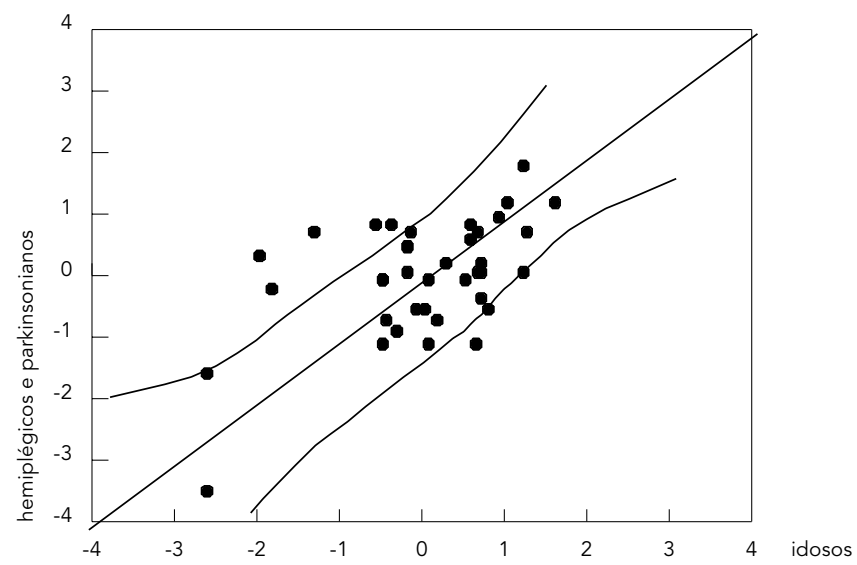

Calibração de itens para idosos sadios versus idosos com incapacidade (hemiplegia e Parkinson). As linhas de controle foram traçadas baseadas no erro associado à calibração de cada item. Itens que caem fora das linhas de controle têm nível de dificuldade diferente.

itens difíceis o suficiente para discriminar indivíduos com uma boa qualidade de vida e, portanto, um escore máximo não significa a melhor qualidade de vida possível para a população estudada, e ainda, os itens mais fáceis são tão fáceis, que não houve indivíduos com uma qualidade de vida tão ruim que não conseguissem realizá-los. Isso indica que talvez essa escala tenha resultados mais expressivos se aplicada a indivíduos mais debilitados, hospitalizados ou institucionalizados, uma vez que mesmo os indivíduos portadores de hemiplegia e doença de Parkinson, avaliados nesse estudo, possuíam um razoável nível de independência funcional. Ressalte-se que esses indivíduos estavam incluídos em programas de treinamento para os quais havia critérios específicos de inclusão, o que de certa forma é uma limitação do estudo, pois dificulta a generalização dos resultados para a população idosa em geral, cuja maioria é representada por indivíduos sedentários. Além disso, o baixo índice de separação $(2,18)$ significa que os itens do perfil dividem as pessoas em apenas cerca de 2,5 níveis de habilidade, e não em três, o número mínimo esperado. Em síntese, a escala é fácil demais e não divide os pacientes em níveis diferenciados de qualidade de vida, discriminando pouco os pacientes.

Para minimizar essas limitações, uma alternativa seria a associação desse tipo de questionário com uma avaliação funcional 5 e uma en- trevista semi-estruturada 30,31 que inclua itens do dia-a-dia do indivíduo, como subir no ônibus, sentar na calçada ou atravessar a rua, e também itens relacionados à parte espiritual.

A inclusão de uma entrevista semi-estruturada pode se tornar uma alternativa importante para incrementar a sensibilidade e a validade do instrumento. Como fonte complementar de dados, uma alternativa viável seria incluir um protocolo de entrevista semi-estruturado com questões mais detalhadas e profundas relativas ao estado atual de qualidade de vida dos indivíduos. Dimensões como atividades de vida diária, cuidado pessoal, segurança, mobilidade pública e atividades gerais de autonomia de vida podem ser obtidas nessa entrevista. As entrevistas devem ser transcritas e analisadas, utilizando procedimentos internacionalmente reconhecidos e métodos atuais utilizados em pesquisas que envolvam análise qualitativa 30 .

Vale ressaltar também a importância de esse tipo de instrumento ser aplicado por um examinador e não auto-administrado, pois, como confirmado pelos dados relativos à escolaridade dos indivíduos entrevistados, a grande maioria tem baixo nível de escolaridade, o que poderia interferir na interpretação das perguntas, comprometendo a fidedignidade dos resultados. Além disso, pudemos detectar através de um estudo piloto que todos os idosos faziam uso de óculos, que nem sempre estavam adequados às suas necessidades e grande parte das vezes, não estavam ao alcance imediato.

\section{Conclusão}

Através da análise Rasch conclui-se que, para os participantes deste estudo, o conjunto de itens que constituem o PSN apresenta validade de constructo, já que apenas dois (5\%) se comportaram como itens erráticos. No entanto, o instrumento apresenta problemas com relação à validade clínica, pois a escala é fácil demais e não mede todo o contínuo de qualidade de vida. Além disso, o questionário discrimina pouco os pacientes, já que divide os indivíduos em apenas dois níveis de habilidade. Isso indica que ele poderia ser mais útil quando aplicado em indivíduos mais debilitados. Para sua aplicação em indivíduos mais capazes funcionalmente, é recomendada a revisão da escala e a inclusão de itens mais difíceis. Portanto, apesar de ser um perfil simples e de fácil utilização, é fundamental a associação desse questionário com uma avaliação funcional e/ou uma entrevista semi-estruturada, de forma a tornar as informações coletadas mais úteis clinicamente. 
Resumo

O objetivo deste estudo foi avaliar as propriedades psicométricas da versão brasileira do Perfil de Saúde de Nottingham (PSN), um instrumento genérico de avaliação da qualidade de vida. O PSN foi traduzido para o português, segundo metodologia recomendada, $e$ aplicado em 215 indivíduos, sendo 170 idosos da comunidade (66,3 $\pm 8,5$ anos), 30 hemiplégicos crônicos (56,4 $\pm 10,9$ anos) e 15 parkinsonianos (59, $6 \pm 10,2$ anos). Os dados foram submetidos à análise Rasch. Dos 38 itens do PSN, apenas dois itens (5\%) não se enquadraram no modelo estatístico, indicando que os itens colaboram para medir um constructo unidimensional. Alguns itens do PSN, no entanto, foram muito fáceis para os idosos, hemiplégicos e parkinsonianos, caracterizando um efeito teto, o que pode limitar o uso da escala para indivíduos com níveis mais altos de habilidade. Apesar dos resultados indicarem que o instrumento se enquadra no modelo estatístico de Rash, há necessidade de revisão dos itens, de forma a tornar o instrumento mais útil clinicamente.

Qualidade de Vida; Perfil de Saúde; Adaptação

\section{Colaboradores}

L. F. Teixeira-Salmela participou da coordenação do projeto, da apresentação do mesmo às agências de fomento, da editoração, da análise estatística e da revisão geral. L. C. Magalhães colaborou na editoração, revisão literária e análise estatística. A. C. Souza e M. C. Lima colaboraram com a coleta dos dados e na elaboração do artigo final. R. C. M. Lima auxiliou na coleta de dados e elaboração do banco de dados. F. Goulart colaboradora do projeto desde o seu início e responsável pelos dados relativos aos indivíduos parkinsonianos.

\section{Agradecimentos}

Ao Conselho Nacional de Desenvolvimento Científico e Tecnológico, Fundação de Amparo à Pesquisa de Minas Gerais e Fundo Fundação de Desenvolvimento da Pesquisa/Universidade Federal de Minas Gerais.

\section{Referências}

1. Berzon RA. Understanding and using health-related quality of life instruments within clinical research studies. In: Staquet MJ, Hays RD, Fayers PM, editors. Quality of life assesment in clinical trials - methods and practice. New York: Oxford University Press; 1998. p. 4-18.

2. Dias RC. Impacto de um protocolo de fisioterapia sobre a qualidade de vida de idosos com osteoartrite de joelhos [Tese de Doutorado]. São Paulo: Escola Paulista de Medicina, Universidade Federal de São Paulo; 1999.

3. Hunt S, McEwen J, McKenna S. Measuring health status: a new tool for clinicians and epidemiologists. Journal of College \& General Practice 1995; 35:185-8.

4. Rejeski WJ, Shumaker S. Knee osteoarthritis and health-related quality of life. Med Sci Sports Exerc 1994; 26:1441-5.

5. Guyatt GH, Feeny DH, Patrick DL. Measuring health-related quality of life. Ann Intern Med 1993; 118:622-9.

6. Kaplan RM. Profile versus utility based measures of outcome for clinical trials. In: Staquet MJ, Hays RD, Fayers PM, editors. Quality of life assessment in clinical trials - methods and practice. New York: Oxford University Press; 1998. p. 69-90.

7. Carr AJ, Thompson PW, Kirwan JR. Quality of life measures. Br J Rheumatol 1996; 36:275-81.

8. Ciconelli RM. Tradução para o português e validação do questionário genérico de avaliação da qualidade de vida "Medical outcomes study 36item short-form health survey (SF-36)" [Tese de Doutorado]. São Paulo: Universidade Federal de São Paulo; 1997.

9. Guillemin F, Bombardier C, Beaton D. Cross-cultural adaptation of health related quality of life measures: literature review and proposed guidelines. J Clin Epidemiol 1993; 46:1417-32.

10. Hunt SM. Cross-cultural issues in the use of quality of life measures in randomized controlled trials. In: Staquet MJ, Hays RD, Fayers PM, editors. Quality of life assesment in clinical trials - methods and practice. New York: Oxford University Press; 1998. p. 51-67.

11. Britto RR, Santos CFF, Bueno FF. Reabilitação pulmonar e qualidade de vida dos pacientes portadores de DPOC. Rev Fisioter Univ São Paulo 2002; 9:9-16.

12. Hunt SM, McKenna SP, McEwen J, Backett EM, Williams J, Papp E. A quantitative approach to perceived health status: a validation study. J Epidemiol Community Health 1980; 34:281-6.

13. Monsó E, Fiz JM, Isquierdo J, Alonso J, Coll R, Rossell A, et al. Quality of life in severe chronic obstrutive pulmonary disease: correlation with lung and muscle function. Respir Med 1998; 92:221-7.

14. Teixeira-Salmela LF, Olney JO, Nadeau S, Brouwer B. Muscle strengthening and physical conditioning to reduce impairment and disability in chronic stroke survivors. Arch Phys Med Rehabil 1999; 80:1211-8.

15. McDowell I. Measuring health: a guide to rating scales and questionnaires. New York: Oxford University Press; 1987. 
16. Haan R, Aaronson N, Limburg M, Hewer R, Crevel H. Measuring quality of life in stroke. Stroke 1993; 24:320-7.

17. Casado J, Gonzalez N, Moraleda S, Orueta R, Carmona J, Gomez-Calcerrada R. Health-related quality of life of elderly patients in primary care. Aten Primaria 2001; 28:167-73.

18. Velozo CA, Magalhães LC, Pan AW, Leitter P. Functional scale discrimination at admission and discharge: Rasch analysis of the level of rehabilitation scale-III. Arch Phys Med Rehabil 1995; 76: 705-12.

19. Goulart F, Teixeira-Salmela LF, Lima O, Moraes S Guedes L, Cardoso F. Physical conditioning and functional performance in Parkinson's disease patients after a physical therapy intervention. Proceedings of the VIIth International Physiotherapy Congress; 2002 Mai 25-28; Sydney, Austrália. Sydney: Australian Physiotherapy Association; 2002. p. 94

20. Morris ME. Movement disorders in people with Parkinson disease: mode for physical therapy. Phys Ther 2000; 80:578-97.

21. Lai JS, Fisher AG, Magalhães LC, Bundy AC. Construct validity of the sensory integration and praxis tests. Occupational Therapy Journal of Research 1996; 16:75-97.

22. Mancini MC, Fiúza PM, Rebelo JM, Magalhães LC, Coelho ZAC, Paixão ML, et al. Comparação do desempenho de atividades funcionais em crianças com desenvolvimento normal e crianças com paralisia cerebral. Arq Neuropsiquiatr 2002; 60 : 446-52.
23. Silverstein B, Fisher WP, Kilgore KM, Harley JP, Harvey RF. Applying psychometric criteria to functional assessment in medical rehabilitation: II - defining inerval measures. Arch Phys Med Rehabil 1992; 73:507-18.

24. Chern J, Kielhofner G, De Las Heras CG, Magalhães LC. The volitional questionnaire: psychometric development and practical use. Am J Occup Ther 1996; 50:516-25.

25. Vieira SC, Mader TH. Avaliação objetiva da performance funcional: desenvolvimento e estudo estatístico [Monografia]. Belo Horizonte: Departamento de Fisioterapia, Universidade Federal de Minas Gerais; 1998.

26. Wright DD, Stone MH. Best test design. Chicago MESA Press; 1979.

27. Linacre MJ, Wright BD. BIGSTEPS: Rasch-model computer program. Chicago: MESA Press; 1992.

28. Wu ZH, Rudkin L. Social contact, socioeconomic status and the health status of the older Malaysians. Gerontologist 2000; 40:228-34.

29. Cutson TM, Laub KC, Schenkman M. Pharmacological and non-pharmacological interventions in treatment of Parkinson's disease. Phys Ther 1995; 75:363-72.

30. Coté J, Salmela JH, Baria A, Russel SJ. Organizing and interpreting unstructured qualitative data The Sport Psychologist 1993; 7:127-37.

31. Osoba D. Guidelines for measuring health quality of life in clinical trials. In: Staquet MJ, Hays RD, Fayers PM, editors. Quality of life assesment in clinical trials - methods and practice. New York: Oxford University Press; 1998. p. 19-35.

Recebido em 26/Mai/2003

Versão final reapresentada em 16/Out/2003

Aprovado em 05/Nov/2003 